\title{
Is blood donation good for the donor?
}

Meyers et al in their article "Possible association of a reduction in cardiovascular events with blood donation" invite us to believe that there is a cause and effect relation between blood donation and a reduction in the risk of subsequent vascular events. First of all, their observational study can, at best, provide evidence only of a statistical link between blood donation and a low risk of vascular events. Whether any confirmed association is cause and effect in nature, a reverse association or owing to inadequate adjustment for unmeasured or unconfirmed confounders, cannot be addressed adequately by their study.

Let us consider the evidence for a statistical association between blood donation and low risk of a vascular event. The crude odds ratio is an impressive $0.5(P<0.001)$. However, this is not interpretable as the donor and nondonor groups are clearly not balanced with respect to a number of major risk factors. Adjusted for these covariates the risk of events in these groups are no longer statistically significantly different. Subsequent subgroup analyses yield only two groups in which nominal statistical significance is achieved after adjustments for covariates that were not balanced in the whole group. However, the upper levels for the associated confidence intervals in both cases were 0.99 , teetering on the brink of non-significance, even if they were the only tests carried out.

After taking into account the multiple subgroup analyses and the fact that the two groups are likely not to be in balance with respect to important unmeasured confounders, and hence are plausibly out of balance with respect to combinations of risk factors or differently out of balance within subgroups (for instance, males and females), the results are far from convincing. Looking at the distribution of the baseline risk factors in donors and non-donors one notes first the imbalance with respect to sex with significantly more donors being male. Despite the over representation of males, a group who would otherwise be at much higher risk, the donors are at lower risk and in fact a number of risk factors were significantly different in the two groups. Other risk factors are surprisingly approximately in balance when pooled across males and females. For instance, high density lipoprotein cholesterol would be expected to be lower in a group dominated by males. This leads one to want to know more about the balance between the two groups for males and females separately. It would not be difficult to raise further questions about the interpretation of these data. Associations identified in observational studies have to be particularly clear cut and convincing, even after adjusting for all potential confounders, before they can be taken seriously.

In summary, the lack of convincing statistical evidence for an association between blood donation and low risk of vascular disease, the possibility of a reverse association in some cases where an early event would discourage subsequent blood donation, and the general perils of observational studies, particularly those containing extensive subgroup analyses, leaves this author, at least, feeling that the interesting and intriguing hypothesis the authors put forward remains just that.

Robertson Centre for Biostatistics,

IAN FORD

Boyd Orr Building,

University of Glasgow,

Glasgow, UK

1 Meyers DG, Strickland D, Maloley PA, Seburg J, Wilson JE, McManus BF. Possible association of a reduction in cardiovascular events with blood donation. Heart 1997;78:188-93. 